\title{
Labeling of groups and events (Terrorism Co-
}

\section{verage)}

\section{AUTHOR}

Liane Rothenberger, Valerie Hase

\section{KEYWORDS}

evaluation, stereotypes, social identity theory, ingroup/out-group

\section{BRIEF DESCRIPTION}

Labeling of groups and events describes how groups connected to religious, political or other forms of violence as well as their acts are labeled or evaluated. These labels might vary from more nominal descriptions (e.g., "gunmen") to more judgmental descriptions (e.g., "terrorist”), leading to different perceptions of these groups and acts by the public.

\section{FIELD OF APPLICATION/THEORETICAL FOUNDATION} Labels for groups and events are of interest in journalism research, political communication, research on terrorism and violence as well as stereotyping. These measurements are often based on "Social Identity Theory" (Brown, 2000) as a theoretical foundation for why some groups and events connected to violence are described in a negative way - i.e., as an out-group -, whilst others are described in a neutral way or even positively, i.e., as an in-group.

\section{REFERENCES/COMBINATION WITH OTHER METHODS OF DATA COLLECTION}

A study by Huff and Kertzer (2017) for example combines a conjoint experiment with an "Automated Content Analysis" of media coverage to understand how the public would label different acts of violence in comparison to the media. Two studies that have been particularly influential in studying the labeling of violent acts and perpetrators will be discussed in more detail in the following sections.

\section{EXAMPLE STUDIES}

Nagar (2010); Weimann (1985)

INFORMATION ON NAGAR, 2010

Authors: Nagar (2010)

Research question: How did American news media cover politically violent organizations that are not linked to Al Qaeda or the events of 9/11?

object of analysis: News coverage by two American newspapers (The New York Times, The Washington Post)

Time frame of analysis: $1998-2004$

\section{INFO ABOUT VARIABLES}

Variable name/definition: Media frame: "First, the labels that describe political violence were coded separately for each segment. Second, the article frame was determined based on the most frequent label." (Nagar, 2010, p. 537)

Level of analysis: Headline, lead paragraph, text Variables and values: four different label categories for labels in text: neutral ("rebel", "rebellion", "insurgent", “insurgency", "guerrilla", "militant", “combatants", "revolt", "uprising", "revolutionary”, "paramilitaries", "insurrection", "separatist"), negative ("terror", "terrorize", "terrorist", "terrorism"), positive ("freedom fighter", "liberation movement", "independence movement"), no label mentioned

Reliability: Krippendorff's alpha: .82

\section{INFORMATION ON WEIMANN, 1985}

Authors: Weimann (1985)

Research question: Which labels did the press use in referring to terrorists when covering terrorist attacks?

Object of analysis: Israel's major newspapers 
Time frame of analysis: 1979-1981

\section{INFO ABOUT VARIABLES}

Variable name/definition: Label

Variables and values: three different labels categories for labels in text: negative ("murderers", "saboteurs", "assassins", "separatists"), neutral ("guerillas", "army”, "front”, "nationalists", "underground", "separatists") and positive ("patriots", "freedom fighters", "liberation movement", "liberation organization")

Reliability: not applicable

\section{REFERENCES}

Boyle, K., \& Mower, J. (2018). Framing terror: A content analysis of media frames used in covering ISIS. Newspaper Research Journal, 39(2), 205-219. doi:10.1177/0739532918775667

Brown, R. (2000). Social identity theory: past achievements, current problems and future challenges. European Journal of Social Psychology, 30(6), 745-778.

De Veen, L., \& Thomas, R. (2020). Shooting for neutrality? Analysing bias in terrorism reports in Dutch newspapers. Media, War \& Conflict. Advance Online Publication. doi:10.1177/1750635220909407

Huff, C., \& Kertzer, J.D. (2017). How the public defines terrorism. American Journal of Political Science, 62(1), 55-71.

doi:10.1111/ajps.12329

Nagar, N. (2010). Who is afraid of the t-word? Labeling terror in the media coverage of political violence before and after 9/11. Studies in Conflict \& Terrorism, 33(6), 533-547. doi:10.1080/10576101003752655

Picard, R. G., \& Adams, P. D. (1987). Characterizations of acts and perpetrators of political violence in three elite U.S. daily newspapers. Political Communication, 4(1), 1-9. doi:10.1080/10584609.1987.9962803

Samuel-Azran, T., Lavie-Dinur, A., \& Karniel, Y. (2015). Narratives used to portray in-group terrorists: A comparative analysis of the Israeli and Norwegian press. Media, War \& Conflict, 8(1), 3-19. doi:10.1177/1750635214531106

Simmons, B. K., \& Lowry, D. N. (1990). Terrorists in the news, as reflected in three news magazines, 1980-1988. Journalism Quarterly, 67(4), 692-696. doi:10.1177/107769909006700423

Weimann, G. (1985). Terrorists or freedom fighters? Labeling terrorism in the Israeli press. Political Communication, 2(4), 433-445. doi:10.1080/10584609.1985.9962776

Table 1. Measurement of "Labeling of Groups and Events" in terrorism coverage.

\begin{tabular}{|c|c|c|c|c|}
\hline Author(s) & Sample & Manifestations & Reliability & Codebook \\
\hline $\begin{array}{l}\text { Boyle \& } \\
\text { Mower } \\
(2018)\end{array}$ & $\begin{array}{l}\text { Newspaper } \\
\text { articles }\end{array}$ & $\begin{array}{l}\text { Computer-assisted key-word } \\
\text { search, looking up labels such as } \\
\text { "terror" }\end{array}$ & $\begin{array}{l}\text { Not } \\
\text { applicable }\end{array}$ & $\begin{array}{l}\text { Not } \\
\text { available }\end{array}$ \\
\hline $\begin{array}{l}\text { De Veen } \\
\text { \& Thomas } \\
(2020)\end{array}$ & $\begin{array}{l}\text { Newspaper } \\
\text { articles }\end{array}$ & $\begin{array}{l}3 \text { different label categories: nega- } \\
\text { tive ("terrorist", "racist", "extre- } \\
\text { mist", "fundamentalist" and clear } \\
\text { links to terrorist organizations } \\
\text { such as ISIS), neutral ("perpe- } \\
\text { trator", "shooter”, "attacker" or } \\
\text { other labels emphasizing race and } \\
\text { ethnicity, for example "Muslim" } \\
\text { or "American"), or positive (fami- } \\
\text { ly- or work-related labels such as } \\
\text { "father" or "colleagues") }\end{array}$ & Not reported & $\begin{array}{l}\text { Not } \\
\text { available }\end{array}$ \\
\hline
\end{tabular}




\begin{tabular}{|c|c|c|c|c|}
\hline Author(s) & Sample & Manifestations & Reliability & Codebook \\
\hline Nagar (2010) & $\begin{array}{l}\text { Newspaper } \\
\text { articles }\end{array}$ & 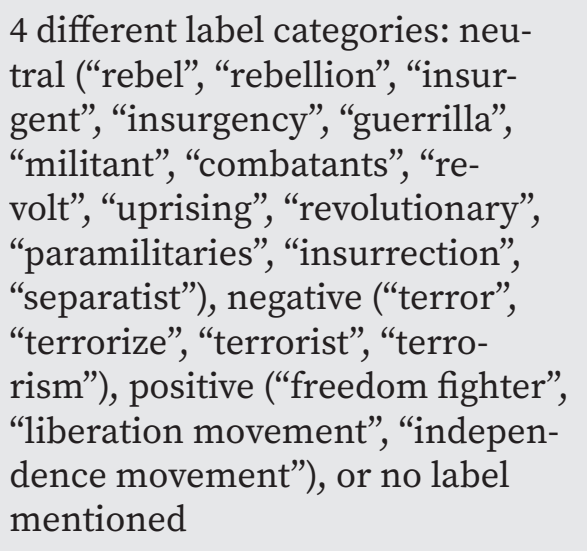 & $\begin{array}{l}\text { Krippen- } \\
\text { dorf's } \\
\text { alpha: .82 }\end{array}$ & $\begin{array}{l}\text { Availa- } \\
\text { ble under } \\
\text { https://www. } \\
\text { hope.uzh.ch/ } \\
\text { doca/article/ } \\
\text { view/2v/1539 }\end{array}$ \\
\hline $\begin{array}{l}\text { Picard \& } \\
\text { Adams (1987) }\end{array}$ & $\begin{array}{l}\text { Newspaper } \\
\text { articles }\end{array}$ & $\begin{array}{l}2 \text { different label categories: nomi- } \\
\text { nal (e.g., "attacker") or descriptive } \\
\text { (e.g., "radical") }\end{array}$ & Holsti: .98 & $\begin{array}{l}\text { Not } \\
\text { available }\end{array}$ \\
\hline $\begin{array}{l}\text { Samuel-Az- } \\
\text { ran et al. } \\
\text { (2015) }\end{array}$ & $\begin{array}{l}\text { Newspaper } \\
\text { articles }\end{array}$ & $\begin{array}{l}7 \text { different labels for perpetrators: } \\
\text { "terrorist/Jewish terrorist", "the } \\
\text { Jewish terrorist", "terror-accused", } \\
\text { "killer", "mass murderer", "serial } \\
\text { stabber/criminal", "other"; } 9 \text { diffe- } \\
\text { rent labels for act: } \\
\text { "terror", "massacre/mass } \\
\text { murders", "bombing/shooting”, } \\
\text { "right wing crime”, "description } \\
\text { assault (stabbing etc.)", "criminal", } \\
\text { "attack", "insanity", "other" }\end{array}$ & $\begin{array}{l}\text { Scott's pi } \\
\text { indicating } \\
\text { lowest value } \\
\text { for any va- } \\
\text { riable in the } \\
\text { study: .86 }\end{array}$ & $\begin{array}{l}\text { Not } \\
\text { available }\end{array}$ \\
\hline $\begin{array}{l}\text { Simmons \& } \\
\text { Lowry (1990) }\end{array}$ & $\begin{array}{l}\text { Magazine } \\
\text { articles }\end{array}$ & $\begin{array}{l}13 \text { different labels for perpetra- } \\
\text { tors: "terrorist", "gunman", "gueril- } \\
\text { la", "attacker", "extremist", "radi- } \\
\text { cal”, "hijacker", "revolutionary", } \\
\text { "nationalist", "armed man/men", } \\
\text { "leftist", "rightist", "militiaman/ } \\
\text { militiamen" }\end{array}$ & Not reported & $\begin{array}{l}\begin{array}{l}\text { Availa- } \\
\text { ble under } \\
\text { https://www. }\end{array} \\
\text { hope.uzh.ch/ } \\
\text { doca/article/ } \\
\text { view/2v/1540 }\end{array}$ \\
\hline $\begin{array}{l}\text { Weimann } \\
\text { (1985) }\end{array}$ & $\begin{array}{l}\text { Newspaper } \\
\text { articles }\end{array}$ & $\begin{array}{l}3 \text { different labels categories for } \\
\text { perpetrators: negative ("murde- } \\
\text { rers", "saboteurs", "assassins", } \\
\text { "separatists"), neutral ("guerillas", } \\
\text { "army”, "front", "nationalists", } \\
\text { "underground", "separatists"), } \\
\text { or positive ("patriots", "freedom } \\
\text { fighters", "liberation movement", } \\
\text { "liberation organization") }\end{array}$ & $\begin{array}{l}\text { Not applica- } \\
\text { ble }\end{array}$ & Not available \\
\hline
\end{tabular}

